cytoskeletal proteins. Indeed, the cytoskeleton is profoundly modified in Alzheimer's disease: microtubules and neurofilaments are greatly reduced in neurons containing paired-helical filaments and tau is expressed in an abnormal phosphorylation state. Alternatively, the degradation system itself could be damaged so that target proteins are improperly ubiquitinated and not recognized by specific proteases, or the proteases themselves could be inactivated. But these hypotheses are still speculative because a ubiquinated protein is not necessarily 'abnormal'. Indeed, ubiquitin has been found bound to proteins not committed to enhanced degradation: ubiquitinated proteins include histones $\mathrm{H} 2 \mathrm{~A}$ and $\mathrm{H} 2 \mathrm{~B}$, actin in Drosophila flight muscle and some membrane receptors. The diversity of the proteins that can be conjugated to ubiquitin indicates that ubiquitination has many cellular functions, but there are few indications of its molecular form in cells and its physiological role in neurodegeneration.

The significance of ubiquitin in degenerating neurons will probably be understood when the ubiquitin moieties in paired-helical filaments and other inclusion bodies are characterized. Once putative protein targets for ubiquitination have been identified, it will be important to determine how these proteins become conjugated to ubiquitin, possibly by studying cellular models expressing the target proteins.

Indeed, ubiquitin is one of the group of proteins called heat-shock proteins which are induced in cells in response to stresses such as thermal shock, heavy metals, oxidants and amino-acid analogues. In conditions of stress, chicken fibroblasts increase their level of ubiquitin conjugates, and during recovery from stress they enhance their overall protein degradation ${ }^{11}$. The study in vivo of the stress response to chronic exposure to toxic agents that induce neuronal degeneration ${ }^{12}$ and ischaemia ${ }^{13}$ may lead to an understanding of the mechanisms of ubiquitin accumulation in inclusion bodies and of its significance in neuronal degenerative diseases.

\footnotetext{
Mori, H et al Science 235, 164I-1644 (1987)

2. Cole, G.M. \& Timiras, P.S. Neurosci. Lett. 79, 207-212 (1987).

3. Perry. G. et al. Proc, natn. Acad. Sci. U.S.A. 84, 3033-3036 (1987).

4. Lennox, G. et al. Neurosci. Lett. 94, 21I-217 (1988) 5. Manctto, V. et al. Proc. natn. Acad. Sci. U.S. A. 85, 4501$4505(1988)$

6. Lowe J et al J Path 155, 9-15 (1988)

7. Leigh, P.N et al Neurosci. Lett 93,197-203 (1988)

8. Lowe, J. ef al. Neurosci. Lett. 94, 203-210 (1988).

9. Murti. K.G. et al. Proc. natn. Acad. Sci. U.S.A. 85, 3019$3023(1988)$

10. Hershko. A. J. biol. Chem. 263, 15237-15240 (1988).

II. Bond U et al J biol Chem. 263, 2384-2388 (1988).

12. Uney, J.B. et al. FEBS Lett. 235, 215-219 (1988)

13. Magnusson. K. \& Wicloch. T. Neurosci. Lett. 96, 264-270 (1989).
}

Jean-Marc Gallo is a CNRS staff scientist currently working with Brian Anderton at St. George's Hospital Medical School, London SWI7ORE, UK

\title{
A nose for Alzheimer's disease?
}

AlzhEIMER's disease is one of several types of dementia. Both for research and for clinical management, it is important to distinguish between the various types, but this can be done with certainty only by postmortem examination of the brain. On page 736 of this issue, B.R. Talamo et al. report observations that may help to resolve this dilemma: in people who died of Alzheimer's disease they find pathological changes in the sensory epithelium of the nose. As this tissue is relatively easy to biopsy, such changes may offer a way of diagnosing the were not, however, seen in olfactory neurons.

The neurons of the olfactory epithelium, which are of central origin, are unusual because they are the only neurons of the mammalian central nervous system known to proliferate throughout adult life (Graziadei, P.P.C. \& Monti Graziadei, G.A. $J$. Neurocytol. $8,1-18 ; 1979)$. This may allow cultures of olfactory neurons to be established in which the molecular changes leading to Alzheimer's disease can be studied directly. The increased phosphorylation of

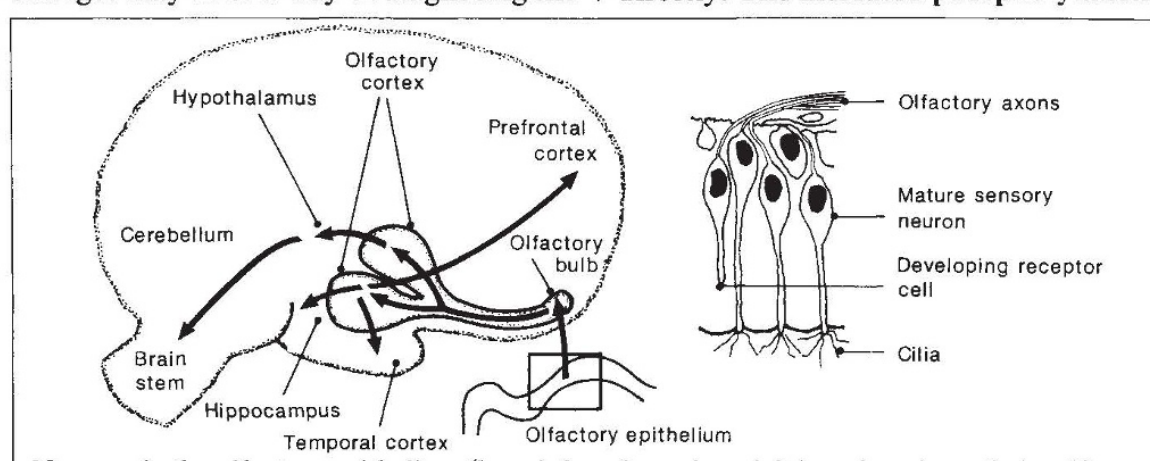

Neurons in the olfactory epithelium (box, left; enlarged on right) project through the olfactory bulb to the olfactory cortex and then to association cortex, hippocampus and hypothalamus, the areas worst affected by the plaques and tangles characteristic of Alzheimer's disease.

disease while the patient is still alive.

Some patients with Alzheimer's disease have both an impaired sense of smell and difficulties with olfactory discriminations. This clue led Talamo and her colleagues to examine the olfactory epithelium (see box) with antibodies specific for proteins known to be involved in the neurofibrillary tangles and neuritic plaques that characterize the brains of Alzheimer's patients. They found abnormal masses of neurites (the axonal and dendritic processes of neurons) in the sensory epithelium of eight out of nine patients who had the typical tangles and plaques in the brain. In contrast, neuritic masses were found in only two out of fourteen controls (aged-matched patients dying of other causes and patients with other neurological disorders).

As well as abnormal morphology, antibody staining revealed a change in the composition of subunits of the neurofilament proteins in the axons of the olfactory neurons. In the normal cases, only the dephosphorylated form of the mid-sized subunit was detected, whereas the Alzheimer's cases also had the phosphorylated form of the heavy subunit, a phosphate-independent form of the mid-sized subunit and the light subunit.

The neuritic masses also reacted strongly with the antibodies for the phosphorylated forms and with two antibodies that specifically stain tangles and plaques in Alzheimer's brains (ALZ50 and a monoclonal antibody to tau, a microtubuleassociated protein found in the paired helical filaments that form a large part of the neurofibrillary tangles). Typical tangles neurofilament proteins, for instance, implies changes in protein kinase or phosphatase regulation. The immaturity of olfactory neurons may make them particularly susceptible to factors such as protease inhibitors, some of which promote neurite outgrowth. One form of the precursor protein of the amyloid protein found in plaques contains a serine protease inhibitorlike insert (see the News and Views article by R. W. Carrell in Nature 331, 478-479; 1988) and glia-derived nexin, which is also a serine protease inhibitor, is found in both the peripheral and central parts of the olfactory pathway (Reinhard, E. et al. Neuron 1, 387-394; 1988).

An outstanding question is how early in the disease these changes can be detected. Clearly, longitudinal studies are now needed as well as a thorough examination of patients with other types of dementia. In Alzheimer's disease, the olfactory regions of the cortex, together with the hippocampus and the olfactory parts of the amygdala, are the worst affected by tangles and plaques. R.C.A. Pearson et al. (Proc. natn. Acad. Sci. U.S.A. 82, 4531-4534; 1988) have suggested that the disease spreads along the projection neurons from the olfactory cortex to the association areas of the temporal, parietal and frontal lobes (see box); the visual, body sensory and motor parts of the cortex are relatively unaffected. It will be fascinating to see whether the disease originates with a malfunction in the way the growth of olfactory neurons is regulated. Jennifer Altman

Jennifer Altman is an assistant editor of Nature. 